Monatsschrift f. Geburtshülfe u. Gynäkologie 1936;101:I-IV

\title{
Contents, Vol. 101, 1936
}

Inhaltsverzeichnis.

Originalarbeiten. Seite

Bak, Michael, Die Wirkung des Schwangerenharnes auf die Entwicklung $\tau$ on Kornfrüchten 45

Borissowa, N. A., Die Beziehungen zwischen dem Gewicht und den

Körpermaßen bei Neugeborenen 66

von Brücke, Hans, Intrauteriner Fruchttod infolge Abschnürung der

Nabelschnur durch das ze/rissene Amnion 16

Charitonow, M. A., und L. S. Kritschewsky, Die veigleichende Bedeutung der Kennzeichen von Hegar und Piseazek in der Diagnostik der frühen Gravidiïät 272

Charischenko, E. J., siehe Schepeiinsky, A. A.

Chmelewsky, W. N., Sohwere Pollakiurie bei Spina biîida occulta, kompliziert durch Retroflexio-versio uteri subfixata 62

Erbslöh, J., Die An wen dung der Bluttransfusion beim Puerperalïieber und bei der Sepsis post abortum 156

Eufinger, H., und Sprado, Gärungshemmende Stoffe im Blut. III. Mitteilung: Das Verhalten gärungshemmender Stoffe im Blut während der normalen und toxischen Schwangerschaft sowie beim Gebärmutterkrebs 5

Fresel, J., Die Lymphe in der Gestation und ihre Beziehung zu eklamptischen Krämpfen 30

Glaeser, siehe Mommsen, H.

Guthmann, Heinrich, und Hildegard Hildebrandí, Über den Einfluß des Geschlechtes des Kindes auf die Entstehung der Schwangersehaftstoxikosen 264

Hildebrandt, Hildegard, siehe Heinrich Guthmann.

Hoffmann, Heinrich, Die Bedeutung der sensiblen Nervendruckreflexe (nach Ferdinand Kehrer, Münster) bei Eklampsie und Präeklampsie 163

Jockel, Hilde, Headsche Zonen während der Geburt, der Schwanger schaft und dem Wochenbett. Beeinflussung der Wehentätigkeit

von den Headschen Zonen aus 249

Koch, Karl, Ein Beitrag zur Pathogenese der intrauterinen Frakturen 11 Kovács, Franz, Über einige seltenere Formen der konsekutiven (sekundären) Gynatresien und Geschlechtskanalverengungen 318

Kritschewsky, L. S., siehe Charitonow, M. A. 
Kritschewsky, L. S., und Erich Werbatus, Eine neue Methode der Be-handlung von Endozervizititen und Erosionen des Uteruskollums mit intrazervikalen Injektionen von Ammoniaksilbersalzlösungen 346

Litwak, B., mxáE.Wiktorowskaja, Regeneration der Uterusschleimhaut nach künstlichem Abort und hysteroskopisches Studium derselben 55 Mommsen, IL, und H. Glaeser, Gärungshemmende Stoffe im Blut. I. Mitteilung: Methodik zum Nachweis gärungshemmender Stoffe im Blut 138

Mommsen, H., und H. Thyssen, Gärungshemmende Stoffe im Blut. II. Mitteilung: Das Verhalten gärungshemmender Stoffe im menstruellen Zyklus und ihre experimentelle Beeinflussung . 257 Nagel, Werner, Über einen Fall primärer Abdominalgravidität mit Sitz in der Milz 23

Naufoks, H., Zur Frage der Strahlenanwendung bei der Sterilisierung aus eugenischen Gründen

$\mathrm{XV}$ Inhaltsverzeichnis.

Seit $\beta$

Pissarczyk, G., siehe Wimhöfer, H.

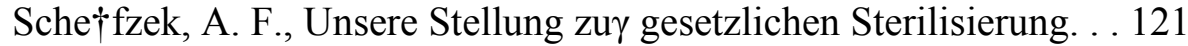

Schepetinsky, A. A., und E. J. Chartschenko, Der Kohlenhydrat- und Phosphorstoffwechsel im Wochenbett und bei puerperalen Erkrankungen 167

Sprado siehe Euiínger, $\mathrm{H}$.

Thiessen, Peter, Utcruszysten als besondere Form der Endometriosen 129

Thyssen, H., siehe Mommsen, H.

Werbatus, Erich, siehe Kritschewsky, L. S.

Wiktorowskaja, E., siehe Litwak, B.

Wimhöfer, H., und G. Pissarczyk, Die Behandlung · der Ovarialinsufiizienz und ihrer Folgeerscheinungen 313

Winkler, Hellmuth, Die Kalium- und Kalziumverschiebung im $\mathrm{g} \cdot$ raviden Uterus. II. Mitteilung: Hire Bedeutung fur den Tonus der Uterusmuskulatur und die Reaktion des graviden Organismus auf Kalziuminjektionen $\quad 34$

Winkler, Hellmuth, Die Veränderungen der Asehenstruktur des infantilen, geschlechtsreiîen und OYulierenden Kaninchenovars. . 141

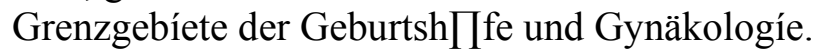

Die Röntgentherapie in der dtynäkologie, Juli 1934 bis Juli 1935.

Von Dr. Hans Bosch-Erlangen 72

Bericht über innersekretorische Literatur (November 1934 bis Oktober 1935). Von Dr. Hans Dorr-Frankfurt a. M 174

Serologie des Karzinoms und der Schwangerschaft. V. Jahresbericht.

Von Dr. Erich Tscherne- Graz 278

Cholezystitis, Cholelithiasis und Appendizitis. Von Dr. Otto Hajek-

Prag 356

Sitzungsberichte aus geburtshilflich-gynäkologischen Gesellschaften. 
Gynäkolog ·ische Gesellschaft zu Breslau. Sitzung vom 12. April 193590 Gesellschaft für Geburtshilfe und Gynäkologie zu Berlin. Sitzungen

vom 24. Mai und 21. Juni 193597

-, Sitzung vom 4. Oktober 1935303

Deutsche Gesellschaft für Gynäkologie. 24. Tagung vom 23. bis

26. Oktober 1935 in München 188,287,363

Gemeinschaftliche Sitzung der Ober-, Mittel- und Niederrneinischen

gynäkologischen Gesellschaft in Bad Kreuznach am 25. und

26. Mai 1935204

Nordwestdeutsche Gesellschaft für Geburtshilfe und Gynäkologie.

Sitzung vom 25. Mai 1935 in Hamburg 106

Bayerische Gesellschaft für Geburtshilfe und Frauenheilkunde.

Bericht über die Tagung am 24. Februar 1935 in München. . 222 Geburtshilîlich-gynäkologische Gesellschaft in Wien. Sitzung vom

12. November 1935244

-, Sitzung vom 10. Dezember 1935307

Buchbesprechungen 115, 246, 309

Personalien und Hochschulnachrichten $\quad 120,309,363$

Professor Dr. Bucura $\dagger \quad 248$

Stephan von Tótk (1865-1935) 310 